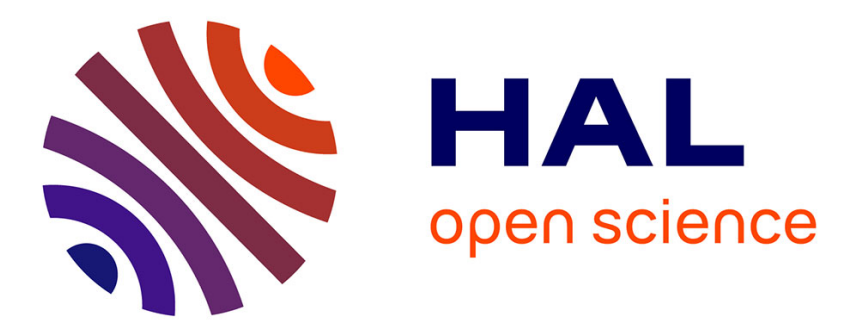

\title{
Self Designing Structures: a new evolutionary rule for thickness distribution in $2 \mathrm{D}$ problems
}

\author{
Gilles Marckmann, P. Bettess, Bernard Peseux
}

\section{To cite this version:}

Gilles Marckmann, P. Bettess, Bernard Peseux. Self Designing Structures: a new evolutionary rule for thickness distribution in 2D problems. Communications in Numerical Methods in Engineering, 2002, 18 (10), pp.743-755. 10.1002/cnm.534 . hal-01007275

\section{HAL Id: hal-01007275 \\ https://hal.science/hal-01007275}

Submitted on 6 Oct 2016

HAL is a multi-disciplinary open access archive for the deposit and dissemination of scientific research documents, whether they are published or not. The documents may come from teaching and research institutions in France or abroad, or from public or private research centers.
L'archive ouverte pluridisciplinaire HAL, est destinée au dépôt et à la diffusion de documents scientifiques de niveau recherche, publiés ou non, émanant des établissements d'enseignement et de recherche français ou étrangers, des laboratoires publics ou privés. 


\title{
Self Designing Structures: a new evolutionary rule for thickness distribution in $2 \mathrm{D}$ problems
}

\author{
G. Marckmann ${ }^{\dagger}$, P. Bettess ${ }^{\ddagger}$ and B. Peseux ${ }^{\dagger}$ \\ † LMM, École Centrale de Nantes, BP 92101, 44321 Nantes cedex 3, F \\ \$ School of Engineering, Durham University, South Road DH1 3LE, UK
}

\begin{abstract}
This paper deals with an evolutionary rule for material distribution in topology optimization problems. This rule is determined in order to satisfy the design constraints that can appear in an industrial design problem. Some other topology optimization techniques are presented before discribing our scheme. The classical MBB problem is used to illustrate the capabilities of our approach. The influences of a design parameter and the mesh refinement are discussed.
\end{abstract}

Key words: topology optimization, self designing structure, evolutionary rule, continuum structure, optimization constraints.

\section{Introduction}

In international competition, manufacturers tend to optimize the cost and the capabilities of their products. The rapid development of sophisticated and inexpensive workstations with graphic capabilities and fast computation has increased the use of computer aided engineering systems. Finite Element Analysis has received wide range attention by companies to validate or improve solutions in mechanical design and many optimization approaches have been integrated into commercial FE programs.

Based on geometry models, shape optimization uses the design variables as the parameters of the geometry to be optimized. Then the best solution with respect to a predefined criterion is found. Nevertheless the selection of the design variables is left to the user. Bakhtiary [1] et al. note that the results of the optimization essentially depend on the number and the selection of the design variables. Anyway, this conventional sizing or shape optimization methods cannot change the structural topology during the solution process so a solution obtained by one of these methods will have the same topology 
as the initial design. This approach is very restrictive, since no hole can be introduced in the design and it cannot lead to solutions where material is used only where it is needed. Moreover, manufacturers tend to reduce the time of conception of their products and shape optimization cannot be used in a phase of pre-study. For this reason, topology or layout optimization methods have received an increasing amount of attention in recent years.

Topology and design optimization consist therefore in determining whether each point of an initial design domain should contain material or not. It gives to manufacturer an idea of what could be the best conception of a part for a given problem.

With the exception of some adaptative techniques, it is customary that approaches to topology optimization use the same finite element mesh to approximate the geometry and the mechanical response fields. Solutions are often rough, and Olhoff and Eschenauer [2] suggest that these methods are most valuable as preprocessing tools for sizing and shape optimization.

Among the survey articles on topology optimization methods, we can cite Rozvany et al. [3] who describe both exact, truss and bars, analytical and approximate approaches and, more recently, Olhoff and Eschenauer [2]. Depending on the type of structure to be considered, two types of topology optimization exist. On the one hand, for inherent discrete structures, the optimum topology problem consists in determining the optimum number, positions and mutual connectivity of the structural members. On the other hand, in topology optimization of continuum structures, the shape of external as well as internal boundaries and the number of inner holes are optimized simultaneously with respect to a predefined design objective.

Our interest is turned towards topology optimization of continuum structures. In this paper, we briefly describe some classical approaches of the problem with their advantages and their drawbacks. Then we will present a simple method based on an engineering approach for 2D structures under plane stress loading.

\section{Topology optimization of continuum structures}

Structural topology optimization is a complex problem. Zhao et al. [4] consider that the lack of exact analytic solutions for response of arbitrary structures, even when composed of simple, linear materials, means that it is not generally possible to obtain an exact solution for the optimal topology of an engineering structure. Nevertheless, from a practical point of view, the problem of design optimization is more a design improvement of an inital mechanical part defined in a finite dimensional design space. This type of structure can be classically 
analysed by a FE approach in order to obtain local or global mechanical information.

Then, the density of material within each finite element is used as a design variable defined between limits 1 (solid material) and 0 (voids or very weak material). The topology optimization problem is formulated as a distributed, discrete value design problem, so called $0-1$ or black\&white problem and illustrated by black and white meshes.

Bakhtiary et al. [1] suggest that two main approaches to the solution of a problem predominate over optimization. The first approach deals with mathematical programming methods. The second one is based on the optimum criteria methods. Reynolds et al. [5] classify them into two main approaches; the method of homogenization and the so-called Element Killing methods.

In this paper, we will briefly present the mathematical concept that leads to direct or relaxed appraoches. The algorithm methods used to solve the problem often depend on the chosen approach.

After the completion of the work described in this paper it was drawn to the authors' attention, by a referee, that a similar approach to that described here, though using a different algorithm, has been adopted independently by Querin et al. [6] and Lencus et al. [7]. Their algorithm was given the name MESO for Morphing Evolutionary Structural Optimisation.

\section{Mathematical concept}

A classical mathematical concept to find the optimal structural topology is to minimize the compliance (or maximize the integral stiffness) of linearly elastic structures under a single or multiple loading condition. The compliance is defined as the work done by the given loading against the displacements at equilibrium, which is equivalent to minimizing the total elastic energy of the structure [2].

$$
\underset{\rho=0 \text { or } 1}{\operatorname{minimize}} \int_{\Omega} F_{V} u \rho d v+\int_{\partial \Omega_{u}} F_{S} u d s
$$

subject to:

$$
\left\{\begin{array}{l}
\int_{\Omega} \delta \varepsilon^{t} E \varepsilon \rho d v-\int_{\Omega} \delta u^{t} F_{V} u \rho d v+\int_{\partial \Omega_{u}} \delta u^{t} F_{S} u d s=0 \quad \forall \delta u^{t} \in U \\
\mathrm{~V}=\int_{\Omega} \rho d v
\end{array}\right.
$$

Here, $\varepsilon$ is the strain field, $E$ the constitutive matrix of the material, $u$ the displacement field, $\delta u$ the virtual displacement field, $U$ the space of kinemati- 
cally admissible trial functions, $F_{V}$ the body force defined on the domain $\Omega$, $F_{S}$ the surface forces defined on the domain boundary $\partial \Omega_{u}$, and $\rho$ the density function. Note that the body force considered here is the weight, then, when the structure is lighted by removing mater, the loading is also removed.

To solve (1), some authors [4], [5] or [2] modify the problem by removing material where it is less useful. The approach leads to such different methods as the Evolutionary Strategy Optimization (ESO) method, the Hard-Kill method or Bubble Method. Criteria or a sensitivity analysis detect parts of the domain to be deleted. They directly tend to solve the $0-1$ problem without modifying it.

It is now well known that the $0-1$ approach is ill-posed in mathematical terms [8] [9] and leads numerically to a lack of convergence. The most usual method to approach (1) is to consider reformulations in terms of continuous variables which allows derivative based optimization methods. This means that one changes the model for material properties to a situation where the volume fraction is allowed to take on any value between zero and one. Despite the improvement in convergence obtained by continuous variables, existence of solutions and mesh dependency may still cause problems, on occasion.

Two similar approaches are reported in the literature to relax the mathematical formulation, such as the homogenization technique or the SIMP algorithm [10]. The first one is the introduction of composites with perforated, periodic microstructures as admissible materials [11]. In the second, one considers an isotropic fictitious material where the stiffness is proportional to the material density of a given solid isotropic reference material [12]. These approach allows the use of derivative based optimization algorithms.

These different techniques are summarised below.

\subsection{Hard-Kill and ESO methods}

In the Hard-Kill method, originated by Hinton [13] [5], elements are gradually removed from the structure by altering their material properties. The criterion for the removal is based on a comparison between the average stress in a given element and the maximum stress in the current design. If the stress is less than a certain fraction (the removal rate decided by the user) of the maximum stress, the element is killed by decreasing the material property to near-zero. To help change at each iteration, the removal rate is increased by a constant amount, also determined by the user, which has the effect of increasing the stress at which elements are killed.

The Evolutionary Structural Optimization (or ESO) method [4] [14], which 
is quite similar to the Hard-Kill method, uses a material efficiency evaluation based on the contribution of an element in the strain energy of the structure. This criterion is used for element removal.

The ESO and Hard Kill methods suffer from some weaknesses [5]. Firstly, the use of two parameters, namely the reject ratio and the evolution rate, set by the user, makes the corresponding solution somewhat arbitrary. The choice of these parameters can be quite problematic, leading to disintegration of the structure if excessively large values are used, or very slow evolutions if small values are used. Secondly, the evolutionary structural optimization method is based on the full stress design concept. This involves the calculation of stresses in finite element and the use of some stress criteria, such as the Von Mises stress or average stress, to determine which element should be removed from the design domain of a structure. Thirdly, an appropriate indicator to be used for assessing the quality of the solution is absent.

\subsection{Bubble method}

There exists a very different, so-called macro-approach to topology design. In the bubble method, the conventional shape design technique is augmented to one of simultaneous topology design by considering additional design variables that govern the number and positions of holes, and where in each step of an iterative hierarchical procedure the introduction and optimum positioning of a new hole is followed by a conventional fixed-topology shape optimization.

Olhoff and Eschenauer [2] note that this method is particularly attractive from the point of view of manufacture, since nice smooth boundaries of the design are obtained and no post-processing is required. Moreover, the complexity of the structure is directly controlled by the user who can avoid less practical solutions.

The bubble method applies mathematical programming, both for formulating the criterion for creating holes, (the Kuhn Tucker conditions), and for optimizing the shape of the holes.

\subsection{Ranked layered material}

In this approach density and orientation are included as admissible designs and their effective mechanical properties can be determined via some sort of homogenization technique [15]. This would mean that theoretically, for each plate element a different material would be used [3] [16]. These microstructure models provide regularization of the topology optimization problem via relax- 
ation of the design space. The results clearly indicate mesh independency and the existence of a well defined limiting design.

These methods, which used a derivative based algorithm, can easily be extended to other kinds of optimization as, for instance, vibration.

But it is a characteristic feature that the structure of optimum topology obtained by this approach generally consists of composite materials in large sub-domains and the introduction of appropriate restrictions is necessary to lead to more distinct black\&white designs which are easier to manufacture.

Methods are introduced to restrict the design space by defining the perimeter of the design as a measure of the boundary of the solid region, by introduction of a local gradient constraint on thickness variation or by filtering techniques that prevent creation of checkerboards in numerical solutions [17]. These three methods produce very similar designs. However, the perimeter method involves a global constraint and will allow the formation of locally thin structural members unlike the other methods. Moreover, the perimeter must be determined by experiments, since there is no direct relation between local scale in the structure and the perimeter bound. In contrast, local filtering corresponds to a lower limit on widths of the structural members and can be defined when manufacturing is taken into consideration.

However, the homogenization technique suffers of the lack of realistic criteria for the stress constraint in material of intermediate density, since the form of the stress constraint is not given a priori [18].

\subsection{SIMP Method}

The SIMP method (Solid Isotropic Material with Penalty) is considered as a generalization of the the variable thickness sheet problem, where the density is interpreted as the thickness function of a two dimensional sheet. This material covers the complete range of density values from 0 to 1 , but does not provide regularization of the classical formulation problem. This drawback disappears if a penalty is included in the formulation. For that, a penalty against intermediate densities is used to approximate the integer problem [19]. The density function enters the stiffness relation via a power $p>1$ which has the effect of penalizing intermediate densities. It is a drawback of the SIMP model that the designs obtained not only exibit dependence on the value of $p$, but also on the finite element mesh applied. Even if such a constraint is not included in the formulation, the SIMP model can yield very nice results, and can, with a simpler formulation, arrive at similar topologies to the microstructure methods. The simplicity of the model greatly facilitates the implementation of topology design in commercial finite element codes. 


\section{An new evolutionary rule for topology optimization in mechanics}

In order to release ourselves from derivative based mathematical programming algorithm we have opted for an evolutionary algorithm. Even if the latter appear less rigorous, in a mathematical sense, they are much less complex and can be easily implemented with a closed FE code where only data file can be modified. Moreover, even if no optimization algorithm is used, they can lead to solutions close to analytically exact solutions or similar to those obtained by mathematical methods.

The introduction of microstrutures leads to a design solution with composite material in large sub-domains, with the exception of the introduction of perimeter method. This latter method is usually programmed in order to obtain a so called back\&white solution for isotropic material.

In our work, we tend to solve problems with isotropic material. Taking into the thickness of the structure $h(x)$, the equation (1) can be reduced in term of thickness, which regularizes the formulation. Then, we always obtain directly a solution with the solid isotropic material which is more useful for the manufacturer.

Unlike some Evolution Strategies (Hard-Kill or ESO), a variable thickness approach has the advantage that no element is removed and a element which makes a small contribution (ie has a small thickness) at a given iteration can be reinforced at another iteration if necessary. This allows back tracking through thickness reductions and iterative correction if necessary.

Even if our approach has high CPU cost dominated by analyse with oscillations around the solution before convergence, it seems to lead to stable solution. Then, the aim of this paper is to validate the thickness evolution method before improving its rapidity.

\subsection{Formulation of the topology problem}

In a first approach, we consider a structure in a plane state of stress under a single load case. The design of the structure has to be determined in a design domain defined by its boundaries. This domain is the portion of the space where the mechanical part can be designed (in the plane but also in thickness). We propose then to improve a first constant thickness structure with an evolutionary rule so that material (thickness) is removed from places where it is not effective. The initial constant thickness is taken as the maximum admissible thickness. 
Removing material (as in the Hard-Kill method, for instance) leads to solutions that tend to disintegrate the structure if the stress constraint is not taken into account. Moreover, this kind of approach is known to be mesh dependent. This means that obtained solution with a certain size of element is different from the one obtained with a finer mesh. Moreover, the stress constraint (for example, the maximum Von Mises stress) is usually useful for the manufacturer for evident reasons.

Nevertheless, we must also consider displacements constraints which can lead, if they are not respected, to an overstepping of operational tolerances.

If the initial problem with a constant thickness already respect the stress and displacement constraints (if they are not respected, the structure cannot be improved), it is always possible to find a better solution for the design problem which satisfies one of the two following equations:

$$
\sigma(x)_{\text {maxi }}=\sigma_{\text {admissible }}
$$

or

$$
u(x)_{\text {maxi }}=u_{\text {admissible }}
$$

These relations can be considered as optimal criteria that the evolution strategy have to reach.

\subsection{Description of the rule}

The observation of the orientation of the principal stresses in an initial constant thickness sheet gives a first estimate of the design we try to obtain. This means that the material has to follow the stress lines and to be concentrated where the stresses inside the structure equilibrate the external forces.

Then the rule which we adopt, is to distribute the thickness inside the sheet as a function of the stress field. A classical approach is the use of the strain energy. In this paper, we propose to choose the distribution of the generalized load $h(x) \sigma(x)$ at the point $x \in \Omega$ as a measure of the practical purpose, where $h(x)$ denotes the local thickness.

In a first step, in order to validate our approach, we use the Von Mises stress 
as a measure to quantify the local loading of the structure:

$$
\left\{\begin{array}{l}
\gamma=\max _{x \in \Omega}\left(\frac{u(x)_{\text {maxi }}}{u_{\text {admissible }}}, \frac{\sigma_{v m}(x)_{\text {maxi }}}{\sigma_{\text {admissible }}}\right) \\
\phi(x)=h_{i}(x) \sigma_{v m}(x) \\
\lambda(x)=\left(\frac{\phi(x)-\phi_{\text {min }}}{\phi_{\text {maxi }}-\phi_{\text {mini }}}\right)^{p} \\
\alpha=\int_{V} h_{i}(x) / \int_{V} \lambda(x) \\
h_{i+1}(x)=\alpha \gamma \lambda(x)
\end{array}\right.
$$

Here, $i$ is the index of iteration, $\gamma$ is a global coefficient that corrects the structure in order to satisfy the global constraints (equations (2)), $\phi$ is the local criterion $\left(\phi_{\text {maxi }}\right.$ and $\phi_{m i n i}$ respectively its maximum and its minimum value), $\lambda$ is the local ratio of thickness and $\alpha$ is a coefficient used to conserve the global stiffness of the structure if $\gamma$ is equal to 1 .

This variable $p$ has the same role as a parameter of contrast in image processing. That means that it smooths or amplifies the thickness gradient in order to favour a variable thickness topology (grey solution) if $p \leq 1$ or a constant thickness topology (black\&white solution) if $p>1$.

The rule described in relations (3) must be completed with two other relations that represent manufacturing constraints and a numerical limit. The first one is that the thickness must not exceed a given thickness (the original thickness), and the second is that the thickness must not be lower that a limit thickness which is so small that element stiffness is insignificant but which is never zero to prevent numerical problems in the Finite Element Analysis (here $h_{m i n i}$ has the same role as $\rho_{\min }$ used by Sigmund and Petersen in [17]). In order to respect the two latter constraints, a predictor-corrector loop is introduced to cut off the lower and the higher values of the thickness function in respect with aimed global stiffness (i.e. $\alpha \gamma$ ). Few iterations are usually necessary to reach the solution.

\subsubsection{About the use of Finite Element}

In order to be able to treat any kind of mechanical problem, we discretize the domain with finite elements. Hinton and Sienz [13] report that, in a hard-kill method (fully stressed design) the checkerboard effect appears in regions with mainly shear stresses.

Sigmund and Petersen [17] discussed about checkerboard effect and suggest the use of high order elements to prevent this effect, for the homogenization 
approach or the SIMP method if the penalization power $p$ is less than 2.29. They also discuss the filter method which makes the design sensibility of a specific element depend on a weighted average over element itself and its direct neighbours. For variable thickness problems, the authors suggest to make sure that finite dimensional space for displacements is sufficiently large compared to the space for density (here thickness) to eliminate checkerboard effect.

To prevent mesh-dependency which is partly due to a bad stress evaluation that leads to stress discontinuity filters, or a smoothing of the discontinuous fields are often used. In our case, we choose to develop the smoothing method. This approach could be criticized, but our intention here is to validate an evolutionary rule, not to discuss stress evaluation.

\section{Examples}

\subsection{Description of the problem}

In order to illustrate the present method, we choose to test it with a classical problem of the MBB beam described in [18]. In this way, comparison with other methods can be made.

The constraints are those used by Duysinx and Bendsøe:

$$
\begin{aligned}
P & =1 \mathrm{~N} \\
\sigma_{\text {admissible }} & =20 \mathrm{~N} / \mathrm{m}^{2}
\end{aligned}
$$

We add the complementary constraints necessary in our scheme:

$$
\begin{aligned}
u_{\text {admis.sible }} & =0.1 \mathrm{~m} \\
h_{\text {maxi }} & =0.5 \mathrm{~m} \\
h_{\text {mini }} & =\frac{h_{\text {maxi }}}{100}
\end{aligned}
$$

The parameter $u_{\text {admissible }}$ is set here so that it is never reached (in order to compare with other methods), and $h_{\max i}$ is set so that the initial constant thickness sheet is compatible with the global constraints (2). This means that we cannot improve a structure which does not satisfy initially these constraints with a constant thickness equal to $h_{\max i}$.

We discretize the domain with quadratic triangular elements for reasons which are independent of the topology problem, and we set the parameter $p$ equal to 2 , in order to find a black\&white solution. 


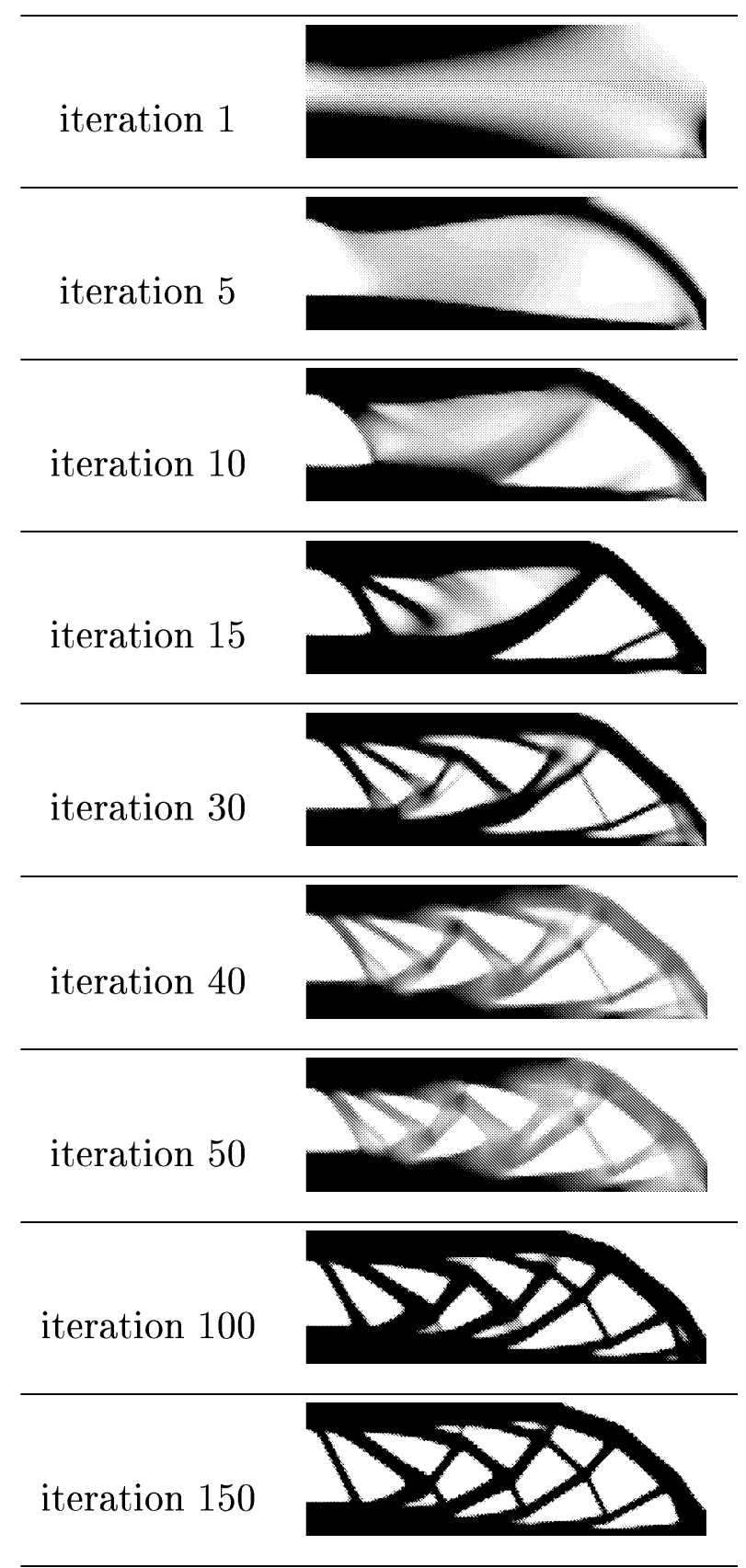

Fig. 1. Evolution of the thickness distribution for mesh composed of 14700 elements T6 and $p=2$

The analytical solution, which is a truss and bar solution, cannot be reached by any optimization method for continuum structures, but the general spirit can be approached. Figure 5.1 shows the evolution of the structures during the procedure. It seems that it could be an interpretation of the solution given by truss and bar method. We can note that the evolution of the thickness distribution is high during the first iterations and it then decreases as the number of the iteration increases till convergence. 


\subsection{Mesh dependency}

Figure 5.2 gives exemples of solutions for different mesh sizes. It shows the solution at iteration 100. The black areas are those where the thickness achieves the upper value. The gray areas are variable thickness zones. It is to note that the iteration 100 is not necessary the converged solution.

It clearly indicates that when the mesh gets finer, the number of members may increase with finer details (see 1200, 2700, 4800 elements) and may change for high refinement with another stronger distibution.

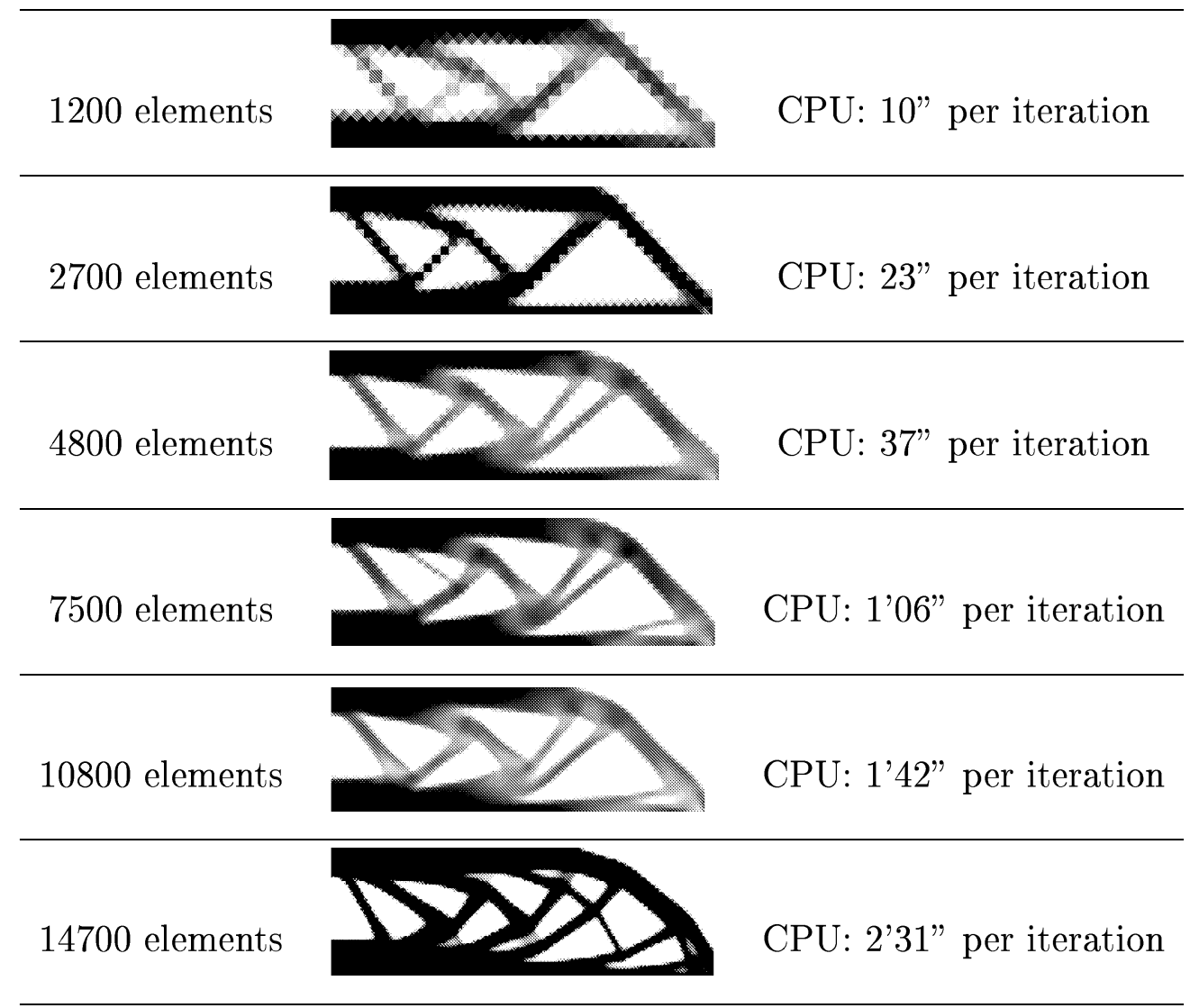

Fig. 2. Mesh dependency on the solution for $p=2$

Appearance of details with the mesh-refinement is often suggested to be consequence of a finer space representation. But it may be also a consequence of the decrease of the stiffness of the numerical model with refinement (or increase of the number of degres of freedom). The decrease of the stiffness leads to a reinforcement of the structure with additional members and then to a change of the topology. This effect appears also with other methods.

This property could be useful for a manufacturer since it could be a way to control the complexity of the structure that he wishes to obtain. 


\subsection{Influence of parameter $p$}

As suggested in the description of the evolution strategy, the parameter $p$ is introduced to help the convergence to a variable thickness plate or to a black\&white solution. The figure 3 illustrates the performance of the method to complete in its ability to reach the kind of structure we wish desire.

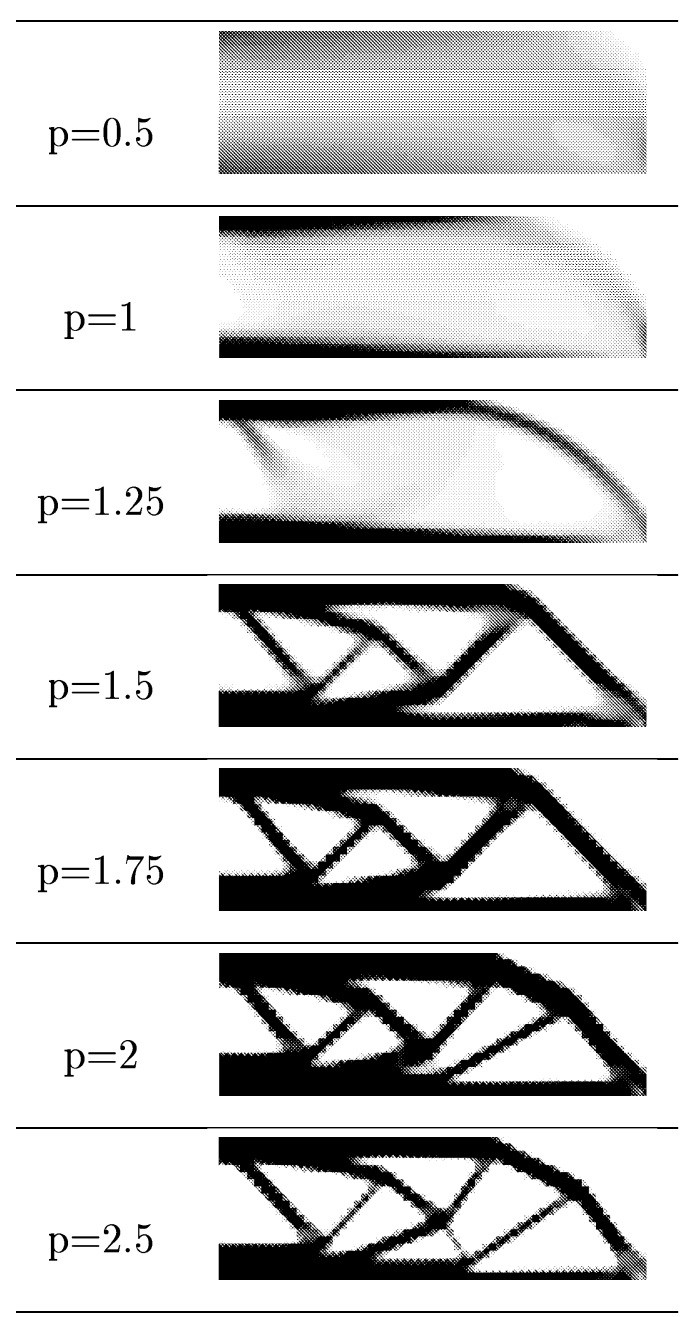

Fig. 3. Influence of the parameter $p$ for a mesh of 4800 elements

This figure clearly shows that the parameter $p$ allows the user to orientate the solution to variable thickness or black\&white solutions. It should be noted that the $p=2.5$ solution is closer to the spirit of the analytical solution. This suggests that there is a optimal value of $p$ that leads to an optimal solution.

Figure 4 illustrated the influence of $p$ on the speed of convergence. For $p<1.25$ convergence is reached with few iterations and there are few oscillations around the solution. But for $1.5<p$ oscillations appear and the amplitude of these oscillations increases with $p$ leading to slow convergences as $p$ increases. 


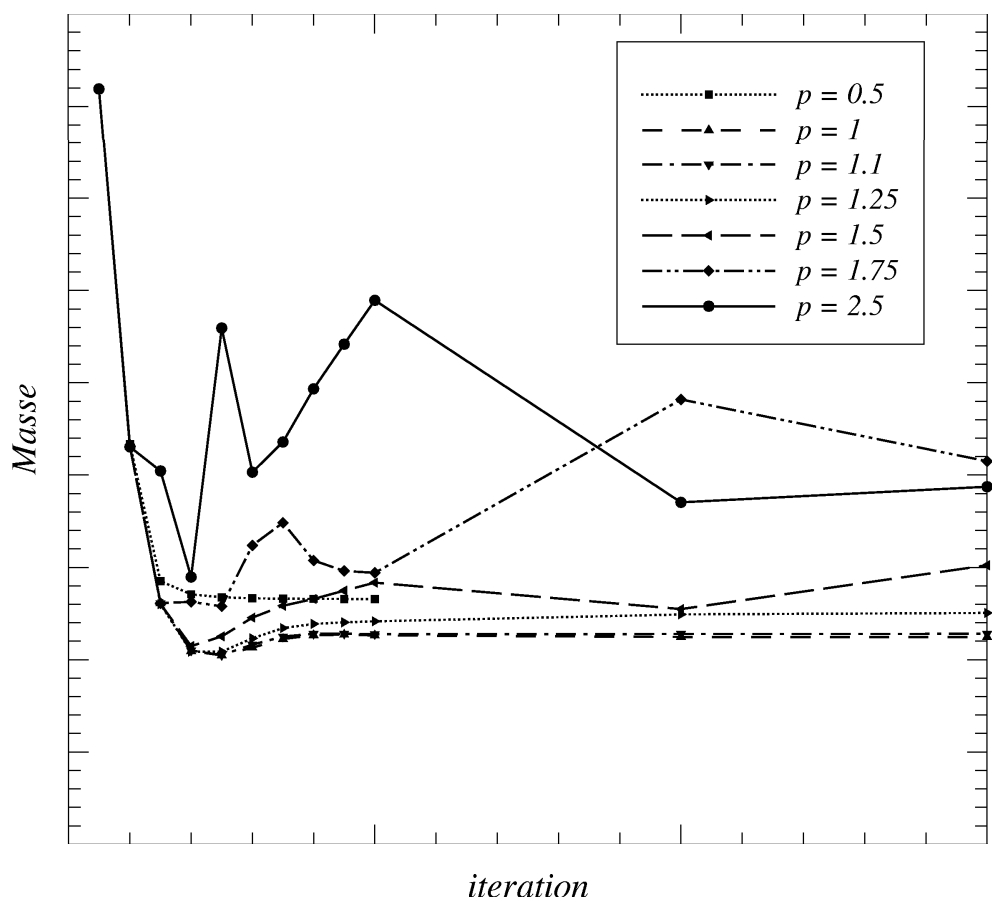

Fig. 4. Evolution of the masse of the structure for different value of $p$

\section{Conclusions and perspectives}

In the first part of this paper, we briefly describe different methods used to solve topology optimization problems. Main advantages and drawbacks are also reported. Then we developed a new approach based on an evolutionary strategy. Taking into account the constraint in displacements is also important, because in some cases, this could lead to different solutions in terms of topology. Based on these optimality criteria, it leads directly to so-called back\&white solutions for appropriate values of the parameter $p$ that satisfy all manufacturing constraints.

This method is successfully used to solve the classical MBB beam problems, with good agreement with analytical or other continuum structures methods.

Then we study influence of the mesh refinement and of the parameter of contrast $p$. This shows that mesh refinement influences the complexity of the solution but the spirit of the analytical solution remains. On the other hand, the parameter $p$ seems to have a optimal value that helps convergence to reach the analytical solution. This simple method, with no mathematical derivative based algorithms, can be easily implemented using a closed FE code for linear elastic 2D problems where only data file is modified.

Now our approach is validated for a single load with some simplification of our inital rule (use of the Von Mises stresses instead of the principal stesses 
or strain energy criteria), it would be interesting to refine our distribution function and, on the other hand, to treat problems with multiple load cases, which are closer to a real industrial case. With our approach, this does not seem to present difficulties. A finer distribution function may also be useful to reduce the number of iterations.

As for all material distribution formulations based on FE analysis, this approach gives a quite rough estimate of the boundary curves of the structure, and a reasonably prediction of the finer details of the boundaries requires the extraction of the new edges obtained with this topology optimization as an initial problem of a shape optimization procedure. This will be also one of our future developments.

\section{References}

[1] N. Bakhtiary, P. Allinger, M. Friedrich, F. Mulfinger, and J. Sauter. A new approach for sizing, shape and topology optimization. In SAE International Congress and Exposition, Detroit, (Michigan USA), February 26-29 1996.

[2] N. Olhoff and H. Eschenauer. On optimum topology design in mechanics. In European Conference on Computational Mechanics, pages 161-231, München (Germany), August 31 - September 31999.

[3] G.I.N. Rozvany, M.P. Bendsøe, and U. Kirsch. Layout optimization of structures. Applied Mechanics Review, 48-2:41-117, 1995.

[4] C. Zhao, P. Hornby, G.P. Steven, and Y.M. Xie. A generalized evolutionary method for numerical topology optimization of structures under static loading conditions. Structural Optimization, 6:250-262, 1993.

[5] D. Reynolds, J. McConnachie, P. Bettess, W.C. Christie, and J.W. Bull. Reverse adaptativity-a new evolutionary tool for structural optimization. International Journal for Numerical Methods in Engineering, 45:529-552, 1999.

[6] O. M. Querin, G. P. Steven, and Y. M. Xie. Morphing evolution structural optimization (meso). In 1st ASMO UK / ISSM conference on Engineering Design Optimization, Ilkley, July 8-9 1999.

[7] A. Lencus, O. M. Querin, G. P. Steven, and Y. M. Xie. Group eso with morphing. In 1st ASMO UK / ISSM conference on Engineering Design Optimization, Ilkley, July 8-9 1999.

[8] G. Strang and R.V. Kohn. Optimal design in elasticity and plasticity. Numerical Methods in Engineering, 22:183-188, 1986.

[9] G. Allaire and R.V. Kohn. Optimal design for minimum weight and compliance in plane stress using extremal microstructures. European Journal of Applied Mechanics, 12:839-878, 1993. 
[10] M.P. Bendsøe. Variable-topology optimization: status and challenges. In European Conference on Computational Mechanics, pages 137-157, München (Germany), August 31 - September 31999.

[11] B. Hassani and E. Hinton. A review of homogenization and topology optimization: I-homogenization theory for media with periodic structure. Computers and Structures, 69:707-717, 1998.

[12] G.I.N. Rozvany, M. Zhou, and T. Birker. Generalized shape optimization without homogenization. Structural Optimization, 4:250-252, 1992.

[13] E. Hinton and J. Sienz. Fully stressed topological design of structures using an evolutonary procedure. Engineering Computations, 12:229-244, 1995.

[14] Y. M. Xie and G. P. Steven. Evolutionary Structural Optimization. Spinger, Berlin, 1997.

[15] M.P. Bendsøe J.M. Guedes, R.B. Haber, P. Petersen, and J.E. Taylor. An analytical model to predict optimal material properties in the context of optimal structural design. Journal of Applied Mechanics, 61:930-937, 1994.

[16] B. Hassani and E. Hinton. A review of homogenization and topology optimization: II-analytical and numerical solution of homogenization equations. Computers and Structures, 69:719-738, 1998.

[17] O. Sigmund and J. Petersson. Numerical instabilities in topology optimization: a survey on procedures dealing with checkerboards, mesh-dependencies and local minima. Structural Optimization, 16:68-75, 1998.

[18] P. Duysinx and M.P. Bendsøe. Topology optimization of continuum structures with local stress constraints. International Journal for Numerical Methods in Engineering, 43:1453-1478, 1998.

[19] W.M. Payten and M. Law. Generalized shape optimization using stress constraints under multiple load cases. Structural Optimization, 15:269-274, 1998. 